\title{
PERCEPCIONES Y EXPERIENCIAS SOBRE LOS DETERMINANTES SOCIALES DE LA SALUD EN UNA MUESTRA DE PROFESIONALES Y ESTUDIANTES DE PSICOLOGÍA Y EDUCACIÓN PARA LA SALUD EN PUERTO RICO \\ PERCEPTION AND EXPERIENCE ABOUT SOCIAL DETERMINANTS OF HEALTH IN A SAMPLE OF PROFESSIONAL AND STUDENTS OF PSYCHOLOGY AND HEALTH EDUCATION IN PUERTO RICO
}

ALÍXIDA RAMOS-PIBERNUS; ELIUT RIVERA-SEGARRA; JOSÉ COLÓN-BURGOS;

OSVALDO GONZÁLEZ-SEPÚLVEDA; CARLOS E. RODRÍGUEZ-DÍAZ; SHEILLA RODRÍGUEZ-MADERA

(UNIVERSIDAD DE PUERTO RICO, Recinto de Ciencias Médicas, Puerto Rico)

\begin{abstract}
RESUMEN
Introducción: Los Determinantes Sociales de la Salud (DSS) son una prioridad para la reducción de las inequidades en salud. La Psicología y la Educación para la Salud son disciplinas cuyo enfoque principal es el mejoramiento de la salud de las personas. No obstante su quehacer tradicionalmente se ha enfocado en el trabajo con los individuos, obviando la atención a los aspectos estructurales. Objetivos: documentar la percepción sobre los DSS en las profesiones de Psicología y en Educación para la Salud en Puerto Rico e identificar experiencias en sus respectivas prácticas con los DSS. Método: Se administró la Encuesta de Percepción sobre Factores Socio-estructurales a 124 profesionales y estudiantes de Psicología y Educación para la salud en Puerto Rico. Para el análisis se utilizaron estadísticas descriptivas. Resultados: se observa un mayor desconocimiento sobre los efectos de DSS como la pobreza, el estigma, el apoyo social y la clase social. Asimismo, la mayor parte de los/as encuestados/as manifestaron que su rol como promotores de la salud se ha centrado a nivel individual fomentando el cambio conductual. Conclusiones: Estos resultados resaltan la importancia de reflexionar críticamente sobre nuestro quehacer y los discursos dominantes en los que nos hemos formado como profesionales de la salud. Se incluyen recomendaciones para trabajar con algunos hallazgos del estudio.
\end{abstract}

PALABRAS CLAVE: Eventos no-epilépticos, disociación somatoforme, inducción de convulsiones, experiencias de abuso infantil, epilepsia. Determinantes Sociales de la Salud, Psicología, Educación para la Salud, formación.

\section{ABSTRACT}

Social Determinants of Health (SDH) have been identified as a priority in the reduction of health inequities. Psychology and Health Education are disciplines that aim to improve the health of people. However, their work has traditionally focused at an individual level, neglecting structural aspects that impact health. The objectives of this study where: (I) document the perception about SDH among Psychology and Health Education professionals in Puerto Rico and (2) identify their experiences in their practices with SDH. The Survey on the Perception about Socio-structural Factors was administered to 124 participants. Descriptive statistics were used for the analysis. Results reflect a greater lack of knowledge on the effects of DSS as poverty, stigma, social support and social class. Also, most of the participants manifested that their role as health professionals has focused at an individual level promoting people to change their behaviors. This results emphasize the importance of critically reflect on our practices and the dominant discourses embedded in us as health professionals. Recommendations for working with the study findings are included and discussed.

KEY WORDS: Social Determinants of Health, Psychology and Health Education

\footnotetext{
Alíxida G. Ramos-Pibernus, Programa de Psicología Clínica, Escuela de Medicina y Ciencias de la Salud de Ponce (PSC/EMCSP); Eliut R. Rivera-Segarra, PSC/EMCSP; José F. ColónBurgos, Escuela Graduada de Salud Pública, Univer sidad de Puerto Rico-Recinto de Ciencias Médicas (EGSP/UPR-RCM); Osvaldo González-Sepúlveda, PSC/EMCSP ; Carlos E. Rodríguez-Díaz, EGSP/UPR-RCM; Sheilla L. Rodríguez-Madera, EGSP/UPR-RCM.

Agradecimientos: Deseamos agradecer a Ashley Díaz, Yarimar Padín, Ednaliz Rosas y Carmen Pérez-Velázquez, quienes contribuyeron en la recopilación de datos en la muestra de Educadores para la Salud.

Correspondencia: Debe ser dirigida a Sheilla Rodríguez Madera al siguiente correo electrónico: sheilla.rodriguez@upr.edu
} 
La Organización Mundial de la Salud (OMS) ha definido la salud como un "estado de completo bienestar físico, mental y social y, no meramente la ausencia de afecciones 0 enfermedades" (OMS, 1946, p. 2). Por diversas circunstancias, no todos los sectores poblacionales gozan de dicho estado por igual. Por ejemplo, la literatura científica ha documentado cómo las tasas de prevalencia de enfermedades y muertes prematuras son mayores en sectores vulnerabilizados socialmente y que los sectores que poseen recursos económicos viven más y tienen un mayor acceso a servicios de salud (Cockerham, 2013; Commission on Social Determinants of Health [CSDH], 2008; Mikkonen \& Raphael, 2010). Estas inequidades tienen un impacto directo en la salud de las personas y son injustas, innecesarias y evitables (Bonnefoy, Morgan, Kelly, Butt, \& Bergman, 2007). La causa de estas inequidades son los determinantes sociales de la salud (DSS; CSDH, 2008; Navarro, 2009). Estos han sido definidos como las circunstancias en las que las personas crecen, viven, trabajan y envejecen; las que a su vez, son provocadas por fuerzas políticas, sociales y económicas (CSDH, 2008; Navarro, 2009).

Los determinantes sociales han sido identificados como una prioridad en la agenda de trabajo para la reducción de las inequidades en salud y la justicia social (OMS, 2008). Desde inicios de milenio la OMS (2003) ha publicado informes para desarrollar consciencia de las implicaciones de determinantes como: el gradiente social, el estrés, la exclusión social, las condiciones en el empleo, el desempleo, la alimentación, las adicciones, el apoyo social, entre otros. De igual forma, se ha señalado la importancia del razonamiento y trabajo interdisciplinario para poder atajar estas inequidades (Venkatapuram \& Marmot, 2009). Sin embargo, el enfoque imperante en algunas profesiones de la salud no ha sido éste. Por el contrario, ha predominado un enfoque intradisciplinario en donde la atención se dirige hacia los aspectos biomédicos e individuales, mientras que los factores socio-estructurales han sido ignorados o relegados a un segundo plano (Raphael, 2006).

Los determinantes sociales como la causa de las inequidades en salud

La literatura sobre DSS establece que los mismos se dividen en determinantes intermediarios y estructurales (CSDH, 2008; Navarro, 2009; Raphael, 2006).

Los determinantes intermediarios y los "factores de riesgo". Según la Comisión sobre los Determinantes Sociales de la Salud, los determinantes intermediarios son aquellos relacionados con aspectos a nivel individual, como conductas y factores fisiológicos, que afectan las diferencias en la exposición y vulnerabilidad a condiciones que comprometen la salud (CSDH, 2008). Algunos de estos determinantes comprenden las circunstancias materiales ( $\mathrm{Ej}$. vivienda 0 capacidad de consumo), el ambiente social o las circunstancias psicosociales (Ej. condiciones laborales 0 apoyo social), factores conductuales o biológicos (Ej. la dieta 0 el consumo de drogas y alcohol), así como el sistema o la estructura que se encarga de la salud y la enfermedad de la población.

Estos determinantes han sido los que más han acaparado el foco de las intervenciones en el área de la salud pública, específicamente los factores conductuales 0 biológicos tradicionalmente conocidos como "factores de riesgo". Este tipo de intervención se ha dirigido al desarrollo de programas de prevención para motivar a las personas a abandonar 0 minimizar las conductas de riesgo (Cockerham, 2013) o a trazar la "causa" de la condición a nivel bacteriológico, genético o molecular (McKinlay \& Marceau, 2000). No obstante, la literatura reciente indica que el trabajo con estos factores intermediarios debe ser contextualizado ya que enfocarse sólo en estos no abona a erradicar las inequidades en la salud que es, en primera instancia, lo que expone a las 
personas al "riesgo de estar en riesgo" (Link \& Phelan, 1995). Para ello, es fundamental tomar en consideración otro tipo de factores conocidos como determinantes estructurales (para efectos de este trabajo utilizamos el concepto estructurantes).

Los determinantes estructurantes y la estratificación social. Los determinantes estructurales son aquellos componentes de la posición socioeconómica de las personas (Ej. pobreza, género, clase social) en conjunto con las condiciones sociales, políticas y económicas (Ej. valores sociales y culturales, políticas públicas y el neoliberalism; CSDH, 2008). Estas condiciones provocan la estratificación social ya que ubican a la población en dichas categorías, las cuales causan las inequidades en salud. Debido a que este proceso de estratificación es uno que se encuentra en constante cambio, para efectos de este trabajo preferimos nombrarles determinantes estructurantes y no estructurales como una manera de recoger la idea del constante movimiento que le caracteriza.

A pesar de que este proceso estructurante es conceptuado como "la causa de las causas" (Marmot, 2006), el paradigma dominante en las estrategias de salud pública continúa siendo el trabajo con los "factores de riesgo" a nivel individual (Raphael, 2006). Esto contrasta significativamente con lo señalado en la literatura sobre la necesidad de enfocarse en los efectos de la política y la economía en la salud para poder atajar las inequidades (Muntaner \& Navarro, 2004). Para ejemplificar lo anterior, tomemos el caso de los Estados Unidos de América donde mueren al año alrededor de 443,000 personas de enfermedades relacionadas al tabaquismo (American Cancer Society, 2012). Los esfuerzos por reducir este hábito se enfocan mayormente en intentar que el individuo voluntariamente evite el uso del tabaco. No obstante, la evidencia científica ha reflejado que este enfoque no es efectivo pues se dejan sin atender factores como las campañas de promoción de compañías transnacionales, los subsidios en los impuestos, los seguros para las siembras así como ayudas en los precios de venta, que deben ser abordados a nivel de políticas públicas (McKinlay \& Marceau, 2004).

Nos parece importante resaltar que aún cuando enfrentamos el reto de un cambio paradigmático, es precisamente en el campo de la salud pública, como disciplina, donde se ha manifestado mayormente el reconocimiento del papel fundamental que ejercen los determinantes sociales en las inequidades en salud. No obstante, sabemos que para lograr implantar una agenda de justicia social y erradicación de las inequidades en salud es imprescindible el trabajo interdisciplinario (Venkatapuram \& Marmot, 2009). Por ello es vital que profesiones de la salud como la Psicología y la Educación en Salud también asuman su responsabilidad ética en esta agenda de trabajo salubrista que pretende contribuir a la erradicación del sufrimiento humano (ArrivillaQuintero, 2009).

El papel de la Psicología y la Educación en Salud en el trabajo con los DSS

Tanto la Psicología como la Educación para la Salud son disciplinas cuya misión es mejorar la salud de las personas (Asociación Americana de Psicología [APA], 2013; OMS, 2013). Sin embargo, aunque la visión sobre cómo abordar dicha empresa difiere en algunos aspectos, su enfoque principal es el trabajo con el individuo y en algunos casos, las comunidades. Veamos el ejemplo de la Psicología.

La APA (2013) define la Psicología como la ciencia que estudia la mente y el comportamiento. Ésta se encarga de estudiar tanto el funcionamiento normal como patológico con el fin de comprender y tratar los problemas mentales, emocionales y conductuales de los individuos. Aunque esta definición evidencia cómo la disciplina se enfoca primordialmente en el individuo como ente aislado, es importante reconocer la 
contribución de sub especialidades como la Psicología Comunitaria. La misma ha tenido un papel central en el abordaje de los aspectos sociales y políticos que afectan la salud de las poblaciones (Montero \& VarasDíaz, 2007). Sin embargo, éste no es el enfoque predominante en la disciplina. $\mathrm{Si}$ tomamos como ejemplo el tema de la obesidad, vemos que la mayor parte de las intervenciones continúan enfocándose en ayudar al manejo de las emociones que puedan vincularse al asunto del sobrepeso y en la adopción de estrategias conductuales tales como: aumento en la actividad física y/o cambios en su dieta (Lindberg \& Stevens, 2007; Martin \& Pear, 1999). No se suelen considerar factores asociados al estado de situación de la seguridad alimentaria en el país o las dificultades que pueda tener la persona en el acceso a alimentos nutritivos.

Por su parte, la Educación para la Salud es una disciplina de la Salud Pública que utiliza la metodología educativa combinada con otras acciones de movilización política, social y organizacional para lograr cambios en los estilos de vida de la población. La OMS (1998) la describe como:

las oportunidades de aprendizaje creadas conscientemente que suponen una forma de comunicación destinada a mejorar la alfabetización sanitaria, incluida la mejora del conocimiento de la población en relación con la salud y el desarrollo de habilidades personales que conduzcan a la salud individual y de la comunidad (p. 13).

Algunos ejemplos de intervenciones son: la educación sexual a personas en riesgo de contraer enfermedades de transmisión sexual, la ayuda a niños y niñas para evitar el uso del tabaco, el alcohol y las drogas, la intervención con personas adultas en el proceso de dejar de fumar, la ayuda a las personas a manejar sus condiciones de salud, así como asistir a las comunidades a organizarse para que éstas sean quienes se encarguen de la mejora de sus condiciones de salud (Glanz, Rimer, \& Viswanath, 2008).
La formación de profesionales de la Psicología, en la mayor parte de los casos, se dirige al trabajo con el individuo como ente aislado (Saforcada, Lellis, \& Mozobancyk, 2010). De igual forma, la Educación para la Salud se ha enfocado en los estilos de vida y los cambios de factores conductuales de la población. Ciertamente al compararse con la Psicología, ésta enfatiza más en el trabajo con las condiciones sociales en general que la primera (UPR-RCM, 2012).

Es evidente que el trabajo para la reducción de las inequidades en salud requiere un cambio en el paradigma para abordar el papel causal de las condiciones sociales en el proceso de salud-enfermedad de las poblaciones. Un paso importante en esta dirección es inspeccionar la ideología subyacente al contexto en donde se manifiestan las inequidades (Muntaner \& Navarro, 2004). La evaluación del contexto político, económico y social de los países permite una mirada al estado actual de los determinantes sociales y cómo estos se han abordado en las diversas disciplinas. En nuestro caso, daremos una mirada panorámica al contexto de Puerto Rico.

\section{Los DSS en el contexto de Puerto Rico}

Puerto Rico es una de las Antillas Mayores ubicada en el Caribe. La Isla mide cerca de 114 millas de largo y unas 42 millas de ancho y, cuenta aproximadamente 3,725,789 personas (Ayuso, Geerman, \& Pesante 2012). El escenario de la Isla es uno complejo ya que nunca ha sido políticamente independiente. Desde 1493 hasta 1898 Puerto Rico fue colonia de España. En el 1898, al finalizar la guerra Hispano Americana, la Isla pasó a ser un territorio no incorporado de los Estados Unidos como botín de guerra (Fernández, 1996). Esto significa que el gobierno norteamericano mantiene control de aspectos como la ciudadanía, el comercio, la migración, las patentes, las comunicaciones, el correo, la transportación, el servicio militar, relaciones internacionales, entre otros (Varas-Díaz \& Serrano-García, 2003). 
El escenario social también es preocupante. Más de 1 millón de la población recibe ayuda del Programa de Asistencia Nutricional, las tasas de desempleo sobrepasan el $14 \%$ y en tan sólo un año se perdieron cerca de 30,000 empleos gubernamentales (Departamento de la Familia, 2007; Departamento del Trabajo, 2013). No es de sorprender que en Puerto Rico, el $45.1 \%$ de la población vive bajo el nivel de pobreza y el ingreso promedio anual ronda en los \$19, 515 (U.S. Census Bureau, 2008-2012). La violencia ha alcanzado niveles epidémicos con cifras de 10,492 crímenes violentos en el año 2009, un $11 \%$ más que el año anterior (Tendenciaspr, 2010). De igual forma, se ha identificado que el abuso de sustancias cuenta con una incidencia poblacional de alrededor de $4.8 \%$. En el área de la salud, las principales causas de muerte en la Isla son las enfermedades cardiovasculares, los tumores malignos (cáncer) y la Diabetes Mellitus (Instituto de Estadísticas de Puerto Rico, 2010). Con respecto a la distribución poblacional por sexo, en Puerto Rico el $52 \%$ de la población son mujeres mientras que el $48 \%$ son hombres (Ayuso, Geerman, \& Pesante, 2012). No obstante, mientras que un hombre jefe de hogar devenga un ingreso anual aproximado de $\$ 29,350$, una mujer jefa de hogar ingresa $\$ 16,389$. Es decir, por ser mujer, el salario es cerca de $\$ 13,000$ menor (Ayuso, Geerman, \& Pesante, 2012). Estas inequidades fomentan que los niveles de pobreza sobrepasen los ya altos niveles identificados para la población general, estimándose específicamente en las mujeres jefas de hogar en cerca de un $58.4 \%$. Todo lo antes expuesto ha redundado en una reducción de la expectativa de vida de la población puertorriqueña que ha sido social y económicamente vulnerabilizada.

Ante un panorama que plantea múltiples retos de índole político, económico y social, junto a sus consecuencias en la salud pública, para efectos de este escrito enfatizamos en varios DSS que a nuestro entender son medulares para la capacitación de profesionales que practican disciplinas inherentes a la Salud Pública. Estos son: pobreza, estigma, apoyo social y la cubierta de salud.

La pobreza. Se estima que cerca de un $45.6 \%$ del total de la población que reside en la Isla se encuentra bajo los niveles de pobreza (Ayuso, Geerman, \& Pesante 2012). Se conoce que la pobreza es uno de los determinantes más importantes que impactan múltiples condiciones de salud (Galindo \& Ardilla, 2012). A medida que el gradiente entre aquellas personas que tienen más recursos y quienes tienen menos se amplía, mayor es la probabilidad de que las últimas se expongan a condiciones que afectan negativamente su salud (Cockerham, 2013; Mikkonen \& Raphael, 2010; OMS, 2003). Por ejemplo, existen estudios en Puerto Rico que apuntan a que cerca del $50 \%$ de la población total presenta inactividad física (Comisión para Evaluar el Sistema de Salud del Estado Libre Asociado de Puerto Rico [CESSELAPR], 2005). Estos datos sugieren una relación en donde a menor ingreso, menor actividad física realizada, lo que a su vez tiene un impacto en la prevalencia de sobrepeso y obesidad en el país.

El estigma. La estigmatización es un proceso en donde se etiquetan las diferencias humanas y se le adscriben características indeseables. Este proceso es contingente del acceso al poder social, económico y político y hace que las personas etiquetadas experimenten el rechazo, la exclusión y la discriminación (Link \& Phelan, 2001). Por tal razón, la literatura reciente ha conceptualizado el estigma como un determinante estructural de la salud (Hatzenbuehler, Phelan, \& Link, 2013; RiveraSegarra \& Ramos-Pibernus, 2013). En Puerto Rico el impacto del estigma en la salud ha sido documentado en diversos grupos poblacionales como por ejemplo, los hombres gays y lesbianas (Toro-Alfonso \& Varas-Díaz, 2004), las personas con problemas de salud mental (Polanco-Fronteras et al., 2013) y las personas con uso de sustancias ilícitas 
(Varas-Díaz, Santiago-Negrón, Neilands, Cintrón-Bou, \& Malavé-Rivera, 2010). En un estudio reciente entre guardias de seguridad y manejadores de caso en el sistema penal de Puerto Rico, se encontraron altos niveles de estigma hacia las personas que viven con $\mathrm{VIH}$ en este contexto (Albizu-García, PérezCardona, \& Santiago-Negrón, 2012). Por otra parte, otros estudios han demostrado cómo profesionales de la salud también mantienen actitudes estigmatizantes hacia las personas que viven con VIH en Puerto Rico. Además, se ha documentado cómo este estigma se encuentra correlacionado con otros como tipos de estigma hacia el uso de sustancias ilícitas y la homofobia (Varas-Díaz, MalavéRivera, \& Cintrón-Bou, 2008). Este escenario presentado en estos estudios ha sido el que ha desencadenado en un provisión de servicios de salud a personas que viven con $\mathrm{VIH}$ de baja calidad e incluso en la negación de los mismos.

El apoyo social. Las personas que no cuentan con apoyo social se encuentran en un mayor riesgo de condiciones de salud crónicas (Ej. Diabetes o Hipertensión; OMS, 2003; Mikkonen \& Raphael, 2010). Lamentable la forma más extrema de falta de apoyo se evidencia en el número de personas sin hogar, que en Puerto Rico ronda los 5,000 (Sermons \& Witte, 2011). La falta de apoyo social se encuentra vinculada con los niveles de pobreza, el género, al igual que el acceso a los servicios de salud (Mikkonen \& Raphael, 2010). La literatura en Puerto Rico refleja cómo la falta de apoyo social perjudica a la población y les pone en mayor riesgo de desarrollar enfermedades. Por ejemplo, estudios realizados han encontrado que el apoyo social es un factor clave en la adherencia a tratamiento antiretroviral en personas con VIH (Nieves-Lugo, \& ToroAlfonso, 2011; Rivera-Rivera, RodríguezMadera, \& Varas-Díaz, 2013). Sin embargo, Varas-Díaz y Toro-Alfonso (2005) exponen que a pesar que de existe evidencia contundente sobre los beneficios del apoyo social en el manejo de enfermedades crónicas, las personas con VIH/SIDA en
Puerto Rico se han visto desprovistas del mismo. Otro estudio que realizó Falcón, Torodova, y Tucker (2009), identificó que los niveles altos de síntomas depresivos en puertorriqueños y puertorriqueñas que viven fuera de Puerto Rico, están asociados a bajos niveles de apoyo social. Estos estudios resaltan el papel del apoyo social como un factor determinante en la salud de la población.

La cubierta de salud. En Puerto Rico la salud no es considerada un derecho. Es por esto que el país cuenta con un sistema de salud privatizado, aunque actualmente se debate la posibilidad de un seguro universal. En este modelo, el Gobierno transfiere a las aseguradoras privadas el manejo del riesgo y son éstas quienes controlan aspectos como la contratación de proveedores, las preautorizaciones, las revisiones de admisiones, entre otros (CESSELAPR, 2005). Se estima que cerca del $57.7 \%$ de la población posee la cubierta pública llamada Mi Salud, mientras que cerca del $8 \%$ no posee cubierta alguna. No obstante, a pesar de estos números, según la CESSELAPR (2005), este sistema no evidencia indicadores en términos de la calidad de los servicios de salud ni en la calidad de vida de las personas. Por ejemplo, se sabe que hay una relación entre la dependencia y el uso de drogas ilícitas con la cubierta de salud ya que la prevalencia es mayor entre quienes se encuentran sin seguro médico o con la cubierta pública (Administración de Servicios de Salud Mental y Contra la Adicción [ASSMCA], 2009).

Como hemos visto, los determinantes sociales como: la pobreza, el estigma, el apoyo social y la cubierta de salud tienen un impacto directo en la salud de puertorriqueños y puertorriqueñas $\mathrm{La}$ literatura nos revela cómo el trabajo primordial se continúa enfocando en el individuo como ente aislado. Es por esto que es vital evaluar cómo disciplinas enfocadas en mejorar la salud de las personas como la Psicología y la Educación en Salud, se insertan en el trabajo salubrista y en la 
reducción de las inequidades en el país. Partiendo del panorama local presentado anteriormente, entendemos que es crucial identificar cuál es la percepción de los profesionales de la salud sobre la interrelación de estas condiciones sociales en el continuo enfermedad/salud para poder trazar estrategias de capacitación adecuadas. A tales efectos, los objetivos de este estudio fueron: (1) documentar la percepción sobre los DSS en las profesiones de la Psicología y la Educación en Salud en Puerto Rico e (2) identificar las experiencias, en sus respectivas prácticas, con los DSS.

\section{MÉTODO}

Para atender los objetivos de este estudio, implantamos un diseño exploratorio transversal utilizando técnicas cuantitativas. Llevamos a cabo este proceso en dos etapas. Durante la primera etapa, llevamos a cabo una revisión de literatura sobre los DSS. En base a esta información, desarrollamos una encuesta para evaluar cuantitativamente las percepciones y prácticas de profesionales y estudiantes graduados de la Psicología y la Educación en Salud con respecto a los DSS. La segunda etapa consistió en la administración de la encuesta. A continuación presentamos una descripción detallada del método implantado.

\section{Participantes}

La muestra estuvo compuesta de 124 participantes (104 mujeres y 20 hombres) entre las edades de 23 a 70 años. Para asegurar que los y las profesionales, así como estudiantes, pudieran consentir legalmente y sin ser presionados, establecimos los siguientes criterios de inclusión: (1) ser mayor de 21 años, (2) encontrarse capaz para consentir, (3) ser estudiante graduado 0 graduada de psicología o poseer licencia de psicólogo 0 psicóloga en Puerto Rico o (4) ser estudiante de Educación en Salud 0 profesional licenciado o licenciada.
Del total de la muestra, 55 personas pertenecían a la disciplina de la Psicología mientras que 69 pertenecían a la disciplina de la Educación en Salud. Todas las personas participantes fueron seleccionadas por disponibilidad. Cada participante recibió una explicación sobre el propósito del estudio, los medios utilizados para proteger la información confidencial y el derecho a culminar su participación en cualquier momento si así lo deseaban. Del total de los/as participantes un $35.5 \%$ reportó ser estudiante al momento del estudio. El grado más alto de escolaridad reportado fue doctorado $(20.2 \%)$ y un $71 \%$ reportó poseer licencia para practicar su profesión en Puerto Rico.

\section{Instrumentos}

Cuestionario sociodemográfico: Desarrollamos el cuestionario sociodemográfico con el propósito de obtener información relacionada con la edad, sexo, ingreso, escolaridad, profesión, religiosidad, entre otras variables que permitieran una descripción de la muestra.

Encuesta de Percepción sobre Factores Socioestructurales: Desarrollamos la encuesta compuesta de 16 reactivos de los cuales 9 evaluaban percepción basada en conocimiento sobre factores socioestructurales y 7 medían la experiencia y exposición de quienes participaron del estudio a los DSS. Los DSS incluidos en la encuesta fueron los siguientes: pobreza, estrés, estigma, apoyo social, seguridad alimentaria, género, clase social, etapas tempranas de desarrollo, e inseguridad en el empleo. Utilizamos dos formatos de respuesta estilo Likert. El primero fluctuaba entre "Totalmente de acuerdo" a "Totalmente en desacuerdo" y el segundo entre "Siempre" a "Nunca".

\section{Procedimiento}

El estudio fue aprobado por el Comité Institucional de Revisión (IRB por sus siglas en inglés) de la Escuela de Medicina y 
Ciencias de la Salud de Ponce protocolo No. 121029SR. Para esto, obtuvimos el permiso y respaldo la Asociación de Psicología de Puerto Rico (APPR) y de la Asociación de Educadores en Salud de Puerto Rico (AESPR). Reclutamos a las personas participantes en dos escenarios: La 59na Convención Anual de la APPR y en la Cumbre Puertorriqueña de Educación en Salud celebradas ambas en el 2012. Como parte del procedimiento de administración, incorporamos herramientas de tecnología móvil (Jones \& Sinclair, 2011). Específicamente, utilizamos el programa computadorizado iSurvey para la administración de la encuesta por medio de iPads. Luego de completar el proceso de reclutamiento de participantes, transferimos los datos al programa SPSS versión 18 para su análisis.

Análisis

Para el análisis de los datos aquí presentados llevamos a cabo estadísticas descriptivas. Específicamente utilizamos medidas de distribución y análisis de frecuencias (Hardy, 2004).

\section{RESULTADOS}

Los resultados que presentamos a continuación, engloban las puntuaciones tanto de estudiantes como de profesionales de acuerdo a la disciplina (Psicología [PSIC] y Educación en Salud [ES]). En términos generales encontramos que la mayoría de los/as participantes mostraron una percepción adecuada basada en conocimiento en premisas relacionadas al papel de determinantes como el estrés, la insatisfacción laboral, la cubierta de salud, y el género en diversas condiciones de salud. Por su parte, determinantes como el estigma, la clase social y el apoyo social no generaron la misma respuesta. En la Tabla 1, presentamos en detalle la frecuencia y el porcentaje de todas las premisas evaluadas.

En la premisa que evalúa cómo perciben el papel del apoyo social en la salud, casi la mitad de las personas participantes $(49.1 \%$ PSIC y $43.5 \%$ ES), reportó no estar de acuerdo en que las personas con escasas redes de apoyo familiar o de pareja presentan altos niveles de incapacidad como resultado de enfermedades crónicas. Cerca de una tercera parte $(29.1 \%$ PSIC y $36.2 \%$ ES) respondió no estar de acuerdo con que la clase social a la que pertenece una persona influye en su salud mental. A su vez, cerca de una cuarta parte $(21.8 \%$ PSIC y $24.6 \%$ ES) estuvo en desacuerdo con la premisa: "El estigma social puede llevar a una persona a tener una muerte prematura". Con respecto al noción de que los individuos tienen la responsabilidad principal de desarrollar las condiciones para mejorar su salud, por encima de la responsabilidad del Estado, la mayoría de las personas participantes estuvieron de acuerdo (78.2\% PSIC y $84.1 \%$ $\mathrm{ES})$.

También realizamos análisis de frecuencia para evaluar la experiencia y exposición de psicólogos/as y educadores/as en salud a los DSS. Pudimos identificar que un $43.6 \%$ de psicólogos/as y un $39.1 \%$ de educadores/as en salud entendía que solamente a veces la pobreza esta relacionada a las condiciones de salud de las personas a quienes proveen servicios. De la misma forma, cerca de la mitad (47.3\% PSIC y $40.6 \%$ ES) reportaron que a veces la inseguridad en el empleo está relacionada a las condiciones de salud de las personas. Al auscultar si incluían en su acercamiento teórico y práctico preceptos provenientes del campo de la Salud Pública, tanto psicólogos/as (63.7\%) como educadores/as (98.6\%) endosaron que siempre lo hacian. Sin embargo la mayor parte de los/as psicólogos/as (72.7\%) y los/as educadores/as en salud (60.9\%) manifestaron que su rol como promotores de la salud se ha centrado a nivel individual fomentando el cambio conductual. La Tabla 2 se enfoca en los aspectos conductuales y muestra en detalle la distribución de frecuencia y porcentajes acerca de la experiencia y exposición de las personas participantes del estudio con respecto a los DSS. 
TABLA 1.

Percepción sobre los Determinantes Sociales de la Salud.

\begin{tabular}{|c|c|c|c|c|c|c|c|c|}
\hline \multirow{3}{*}{ Pregunta } & \multicolumn{4}{|c|}{ Psicólogos/asa } & \multicolumn{4}{|c|}{ Educadores/as para la Saludb } \\
\hline & \multicolumn{2}{|c|}{ De acuerdo } & \multicolumn{2}{|c|}{ En desacuerdo } & \multicolumn{2}{|c|}{ De acuerdo } & \multicolumn{2}{|c|}{ En desacuerdo } \\
\hline & $n$ & $\%$ & $\mathrm{n}$ & $\%$ & $\mathrm{n}$ & $\%$ & $\mathrm{n}$ & $\%$ \\
\hline $\begin{array}{l}\text { 1.Una persona que frecuentemente se enfrenta a momentos } \\
\text { de tensión, se expone a una serie de condiciones de salud } \\
\text { como infecciones, diabetes y accidentes cardiovasculares. }\end{array}$ & 50 & 90.0 & 5 & 9.1 & 58 & 84.1 & 11 & 15.9 \\
\hline $\begin{array}{l}\text { 2. El estigma social puede llevar a una persona a tener una } \\
\text { muerte prematura }\end{array}$ & 43 & 78.2 & 12 & 21.8 & 52 & 75.4 & 24.6 & 17 \\
\hline $\begin{array}{l}\text { 3. Una persona insatisfecha con su trabajo puede desarrollar } \\
\text { dolores en la espalda baja y enfermedades cardiovasculares. }\end{array}$ & 53 & 96.4 & 2 & 3.6 & 65 & 94.2 & 4 & 5.7 \\
\hline $\begin{array}{l}\text { 4. Las personas con escasa redes de apoyo familiar y/o de } \\
\text { pareja presentan altos niveles de incapacidad como resultado } \\
\text { de enfermedades crónicas. }\end{array}$ & 28 & 50.9 & 27 & 49.1 & 39 & 56.5 & 30 & 43.5 \\
\hline $\begin{array}{l}\text { desarrollar las condiciones para mejorar su salud, por encima } \\
\text { de la responsabilidad del Estado. }\end{array}$ & 43 & 78.2 & 12 & 21.9 & 57 & 84.1 & 12 & 15.9 \\
\hline $\begin{array}{l}\text { 6. La prognosis de las condiciones que afectan a las } \\
\text { personas depende en gran medida de su cubierta de salud. }\end{array}$ & 45 & 81.8 & 10 & 18.2 & 52 & 75.5 & 17 & 24.6 \\
\hline $\begin{array}{l}\text { 7. El vínculo entre la obesidad y el bajo nivel socioeconómico } \\
\text { es inexisten. }\end{array}$ & 5 & 9.1 & 50 & 90.9 & 11 & 19.9 & 58 & 84.1 \\
\hline $\begin{array}{l}\text { 8. Los hombres tienen una mayor expectativa de vida que las } \\
\text { mujeres. }\end{array}$ & 6 & 10.9 & 49 & 89.1 & 11 & 19.9 & 58 & 84.1 \\
\hline $\begin{array}{l}\text { 9. La clase social a la que pertenece una persona influye en } \\
\text { su salud mental. }\end{array}$ & 39 & 70.9 & 16 & 29.1 & 44 & 63.8 & 25 & 36.2 \\
\hline
\end{tabular}

Nota. ${ }^{a} n=55 .{ }^{b} n=69$.

TABLA 2.

Experiencia y exposición a Determinantes Sociales de la salud

\begin{tabular}{cccccccccccc} 
& \multicolumn{4}{c}{ Psicólogos/as } & & \multicolumn{4}{c}{ Educadores/as para la Salud } \\
\hline \multicolumn{2}{c}{ Siempre } & \multicolumn{2}{c}{ A veces } & \multicolumn{1}{c}{ Nunca } & \multicolumn{2}{c}{ Siempre } & A veces & \multicolumn{2}{c}{ Nunca } \\
\hline $\mathrm{n}$ & $\%$ & $\mathrm{n}$ & $\%$ & $\mathrm{n}$ & $\%$ & $\mathrm{n}$ & $\%$ & $\mathrm{n}$ & $\%$ & $\mathrm{n}$ & $\%$ \\
\hline
\end{tabular}

1. La pobreza se relaciona con condiciones de salud en las personas a quienes proveo servicios.

$\begin{array}{llllllllllll}27 & 49.1 & 24 & 43.6 & 4 & 7.3 & 36 & 52.2 & 27 & 39.1 & 6 & 8.6\end{array}$

2. Las características afectivas y de cuidado durante etapas

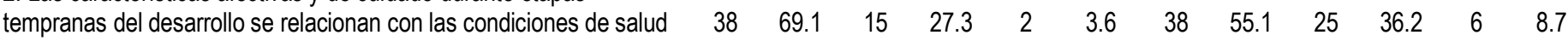
en las personas a quienes proveo servicios.

3. La inseguridad en el empleo se relaciona con las condiciones de salud en las personas a quienes proveo servicios.

4. La disparidad social que se genera a partir de nuestro sistema económico influye en patrones de conductas adictivas (Ej: tabaco, alcohol, drogas ilegales) en personas a quienes proveo servicios.

5. Como profesional, incluyo en mi acercamiento teórico y práctico, preceptos que provienen del campo de salud pública.

6. Mi rol como promotor/a de la salud se ha centrado en el nivel individual fomentando el cambio conductual en las personas a quienes proveo servicios

7. El tema de los determinantes sociales de la salud ha sido discutido como parte de los cursos graduados que he tomado.

$\begin{array}{lllllllllll}40 & 72.7 & 13 & 23.6 & 2 & 3.6 & 42 & 60.9 & 25 & 36.2 & 2\end{array}$

$\begin{array}{llllllllllll}24 & 43.6 & 17 & 30.9 & 14 & 25.5 & 43 & 62.3 & 21 & 30.4 & 5 & 7.2\end{array}$ 


\section{DISCUSIÓN}

De acuerdo a la información obtenida, la mayor parte de las personas participantes reportó un conocimiento adecuado sobre algunos DSS e indicaron poder identificar la presencia de los mismos en las personas a quienes proveen servicios. De igual forma, indicaron incluir en su acercamiento teórico y práctico preceptos de la Salud Pública.

Entendemos que es importante preguntarnos: ¿Qué significa que las personas participantes adjudiquen la responsabilidad de su salud al individuo por encima del Estado? ¿Cuál es el hilo conductor que puede aportar al entendimiento de por qué hay un menor reconocimiento del papel que tienen la clase social, el estigma y el apoyo social en la salud?.

Desde nuestra perspectiva las respuestas a estas interrogantes guardan relación con las políticas neoliberales que han prevalecido en nuestro contexto desde la década de los 80 , cuyos efectos se han materializado en el ámbito de la salud. Estas políticas se sostienen en premisas que apoyan que el Estado reduzca sus intervenciones en las actividades económicas y sociales y, la desregulación de los mercados para potenciar sus posibilidades. Esta ideología favorece a las clases dominantes y sus efectos en el ámbito de la salud son evidentes: la privatización de los seguros de salud, beneficiando a sectores con alto ingreso económico a expensas de las clases populares (Navarro, 2009). La ideología neoliberal se evidencia en el paradigma que ha dominado la Salud Pública desde las pasadas décadas, el cual enfoca en una plétora de acciones que tiene que realizar el individuo para "estar saludable" (i.e. cambios en estilos de vida) minimizando así la responsabilidad que tiene el Estado de proveer el andamiaje necesario para este fin.

Tomando en cuenta que la mayor parte de las personas participantes atribuyen la responsabilidad de desarrollar las condiciones para mejorar o empeorar las condiciones de salud al individuo, y que igualmente señalan que su rol como promotoras de la salud se enfoca en el cambio conductual; nos parece que estas respuestas son cónsonas con el discurso de sus respectivas disciplinas y con los postulados del paradigma actual en la Salud Pública que enfatiza en "factores de riesgo" y estilos de vida. Sin embargo, estos datos no van a la par con lo evidenciado en la literatura sobre los DSS la cual ha documentado la necesidad de trascender este enfoque individual y atender los efectos de las políticas del Estado en la salud de las poblaciones (McKinlay \& Marceau, 2004; Muntaner \& Navarro, 2004).

Así las cosas, los psicólogos/as y educadores/as para la salud en nuestro contexto son educados para promover cambios conductuales, en la mayoría de una manera descontextualizada del entorno socioestructural del individuo. El efecto directo de lo anterior se refleja en una falta de entendimiento por parte de profesionales de la salud sobre las consecuencias de las inequidades en la salud pública, especialmente en torno a determinantes claves como son la clase social y el estigma social.

La falta de reconocimiento del papel que ejerce la clase social en la salud evidenciada en las personas participantes se puede explicar a la luz de lo que plantea Navarro:

El análisis de clase es frecuentemente descartado como uno anticuado, un tipo de análisis y discurso de "ideólogos", no para científicos rigurosos y serios. El concepto de clase, prácticamente ha desaparecido de la literatura científica y se ha sustituido por "estatus" u otras categorías menos conflictivas. La desaparición del análisis de clase y el discurso de clase, sin embargo, tiene una motivación política. Es precisamente un signo del poder de clase (el poder de la clase dominante) 
que el análisis de clase haya sido sustituido por categorías de análisis menos amenazantes para el orden social. En este nuevo escenario, la mayoría de los ciudadanos se definen como clase media, la gran mayoría de las personas que se coloca entre "los ricos" y "pobres" (2009; p. 7).

Tal pareciera que el concepto de clase social en nuestro momento de época se torna "políticamente incorrecto" o como establece Navarro (2009), el mismo ha ido desapareciendo del discurso político y científico, aún cuando las tensiones aumentan cada día producto de la inequidad.

Por su parte el hallazgo sobre el estigma como determinante social no debe sorprendernos. Sabemos que el estigma es un mecanismo precursor de la exclusión social. De igual forma se conoce que la exclusión social disminuye la expectativa de vida (Wilkinson \& Marmot, 2003). El mismo está institucionalizado y legitimado mediante políticas públicas (Varas-Díaz, MalavéRivera, \& Cintrón Bou, 2008) y afecta especialmente a sectores que han sido identificados como "no gratos" socialmente como son las personas de las comunidades LGBTT, con VIH, con uso problemático de sustancias, inmigrantes, trabajadoras sexuales, entre otros. En Puerto Rico existen estudios que documentan altos niveles de estigma en profesionales de la salud (Polanco-Fronteras et al. 2013; Varas-Díaz, Malavé-Rivera, \& Cintrón-Bou, 2008; VarasDíaz, Santiago-Negrón, Neilands, CintrónBou, \& Malavé-Rivera, 2010). Lo anterior puede apuntar a la falta de adiestramiento e intervenciones dirigidas al manejo de estigma entre profesionales de la salud. Los altos niveles de estigma podrían ser un reflejo de la falta de conocimiento de cómo este fenómeno se manifiesta y el impacto negativo que tiene para la salud pública y los servicios de cuidado.

Como se observa en los datos de este estudio, los y las participantes subestimaron el efecto positivo del apoyo social para la salud. Este dato no es congruente con lo reflejado en la literatura en torno al hecho de que las personas que cuentan con poco 0 ningún apoyo social se encuentran en un mayor riesgo de desarrollar condiciones de salud crónicas (OMS, 2003; Mikkonen \& Raphael, 2010). Esta dimensión protectiva del apoyo social no solo debe evaluarse en sus efectos a nivel individual, sino que es facilitador de la cohesión social. Nos referimos a las palabras de Muntaner y Lynch (2002) para aportar al entendimiento de este hallazgo:

Las clases y sociedades raciales, donde el bienestar de algunos depende de la privación relativa de los demás es políticamente inestable y tarde 0 temprano generan conflictos... Sólo hay una cantidad limitada de cohesión que las clases trabajadoras y los géneros subordinados o grupos raciales/étnicos tolerarán antes de participar en la disidencia política con los propietarios ricos o de género dominante o grupos raciales/étnicos (p. 266).

Entendemos que es fundamental reflexionar críticamente sobre nuestro quehacer y los discursos dominantes en los que nos hemos formado como profesionales de la salud. A continuación incluimos varias recomendaciones para trabajar con algunos de los hallazgos del estudio y que además, facilitarían la transición a un modelo de Salud Pública que considere los determinantes sociales de la salud. Todas las recomendaciones presentadas se aplican a las diferentes profesiones relacionadas a la provisión de servicios de salud, incluyendo la Psicología y la Educación para la Salud. Los dividimos en las siguientes áreas: (1) formación profesional, (2) el estigma, y (3) el apoyo social. 
Formación profesional

1. Revisar los currículos de enseñanza para incluir un acercamiento a la salud desde los determinantes sociales.

2. Asegurar que nuestras intervenciones reflejen una comprensión del papel de los determinantes sociales.

3. Promover que las organizaciones que agrupan profesionales de la salud promuevan los preceptos implicados en el modelo de DSS.

4. Fomentar el mejoramiento de ambientes educativos, laborales y comunitarios que promuevan el apoyo y la cohesión social.

5. Trabajar las condiciones de salud desde un acercamiento interdisciplinario.

\section{Estigma}

1. Adiestrar sobre el sobre el manejo del mismo en profesionales de la salud.

2. Eliminar políticas públicas estigmatizantes.

3. Continuar el desarrollo de investigaciones dirigidas a identificar cómo el estigma opera en la salud.

Apoyo social

1. Participar en el desarrollo de políticas dirigidas a la erradicación de inequidades económicas y la exclusión social.

2. Recibir adiestramientos sobre temas que permitan conceptuar el papel de economía política de la salud.

3. Fomentar espacios de cohesión social.

4. Diseñar áreas físicas que promuevan la interacción social.

5. Mejorar ambientes educativos, laborales y comunitarios para proveer apoyo (social, emocional, labores/tareas).

Los hallazgos aquí compartidos, aunque surgen de un esfuerzo investigativo que enfoca en profesionales y estudiantes de la Psicología y la Educación para la Salud en el contexto de Puerto Rico, tienen pertinencia para otros contextos dado que proveen información valiosa para el análisis sobre las implicaciones del paradigma dominante en Salud Pública en la formación de profesionales de la salud. El discurso de la llamada "nueva salud pública" ha proliferado marcadamente durante las pasadas dos décadas resultando en políticas que enfatizan en el autocuidado; restándole al Estado la responsabilidad que tiene en este haber (Petersen \& Lupton, 1996). El impacto de este acercamiento es preocupante si tomamos en cuenta que la salud lejos de verse como un derecho, se trata como un negocio, a juzgar por la forma en que operan los seguros de salud. 


\section{REFERENCIAS}

Alessi, Vincentiis, Rzezak y Valente (2013). Semiology of psychogenic nonepileptic seizures: Age-related differences. Epilepsy \& Behavior, 27, 292-295.

Administración de Servicios de Salud Mental y Contra la Adicción. (2009). Trastornos de Sustancias y Uso de Servicios en Puerto Rico. Puerto Rico: autor.

Albizu-García, C., Pérez-Cardona, C. \& Santiago-Negrón, S. (2012). Correlates of HIV Stigma in prison guards and case workers in a national prison system. Retrovirology, 9(Suppl. 1), 67.

American Cancer Society. (2012). El hábito de fumar cigarrillos. Recuperado de http://www.cancer.org/acs/groups/cid/docu ments/webcontent/002966-pdf.pdf

American Psychological Association. (2013). How does the APA define "psychology"? http://www.apa.org/support/about/apa/psyc hology.aspx\#answer

Arrivilla-Quintero, M. (2009). Psicología y salud pública. Universitas Psychologica, 8(1), 137-147.

Ayuso, I., Geerman, K., \& Pesante, F. (2012). Puerto Rico Community Health Assesment:

Secondary Data Profile. Departamento de Salud Gobierno de Puerto Rico.

Bonnefoy, J., Morgan, A., Kely, M.P., Butt, J., \& Bergman, V. (2007). Constructing the evidence base on the social determinants of health: A guide.

Central Intelligence Agency. (2012). The World Factbook. United States: Author. https://www.cia.gov/library/publications/theworld-factbook/geos $/ \mathrm{rq}$.html

Cockerham, W. (2013). Social Causes of Health and Disease. Great Britan: Polity Press.

Comisión para Evaluar el Sistema de Salud del Estado Libre Asociado de Puerto Rico (2005).

Evaluación del Sistema de Salud de Puerto Rico: Hacia el desarrollo integral del sistema de salud de Puerto Rico, ciudadanos de conciencia salubrista y un sistema de salud con perspectiva intersectorial, holística, emancipadora, proactiva y a base del derecho

humano a la salud. Boletín Administrativo, Núm. OE-2005-13.

Commission on Social Determinants of Health. (2008). A Conceptual Framework for Action on the Social Determinants of Health. World Health Organization.

Departamento de la Famila. (2007, Octubre). Compendio de Estadísticas Sociales 20022003 al 2005-2006. Estado Libre Asociado de Puerto Rico.

Departamento del Trabajo y Recursos Humanos. (2013, abril). Empleo y desempleo Puerto Rico. Negociado de Estadísticas del Trabajo, Estado Libre Asociado de Puerto Rico.

Falcón, L. M., Todorova, I., \& Tucker, K. (2009). Social support, life events, and psychological distress among the Puerto Rican population in the Boston area of the United States. Aging \& Mental Health, 13(6), 863-873. doi:10.1080/13607860903046552

Fernández, R. (1996). The Disenchanted Island: Puerto Rico and the United States in the Twentieth Century. USA: Praeger.

Galindo, O., \& Ardilla, R. (2012). Psicología y pobreza. Avances en Psicología Latinoamericana, 30(2), 381-407.

Glanz, K., Rimer, B., \& Viswanath, K. (2008). The Scope of Health Behavior and Health Education. In K.Glanz, B. Rimer \& K Viswanath (Eds). Health Behavior and Health Education: Theory, Research and Practice. (4th Ed). San Francisco: JosseyBass.

Hardy, M. (2004). Summarizing distributions. In M. Hardy \& A. Bryman (Eds.). Handbook of data analysis. London: SAGE publications Ltd.

Hatzenbuehler, M., Phelan, J. \& Link, B. (2013). Stigma as a Fundamental Cause of

Population Health Inequalities. American Journal of Public Health, 103 (5), 813-821.

Instituto de Estadísticas de Puerto Rico (2010). Nuevas estadísticas de mortalidad, 20002008. San Juan, Puerto Rico. Recuperado de www.estadisticas.gobierno.pr.

Jones, J. L., \& Sinclair, B. (2011). Assessment on the Go: Surveying Students With an iPad. Journal of Library Innovation, 2(2), 22-35.

Lindberg, N. M., \& Stevens, V. J. (2007). Review: weight-loss interventions with Hispanic populations. Ethnicity and Disease, 17(2), 397-402.

Link, B., \& Phelan, J. (1995). Social Conditions as Fundamental Causes of Disease. 
Journal of Health and Social Behavior, 35, 80-94.

Link, B. \& Phelan, J. (2001). Conceptualizing Stigma. Annual Review of Sociology, 27, 363-385.

Marmot, M. (2006). Introduction. In M. Marmot \& R. Wilkinson (Eds.). Social Determinants

of Health. (2nd Ed). New York: Oxford University Press.

Martin, G., \& Pear, P. (1999). Behavior Modification: What it is and how to do it. New Jersey: Prentice-Hall, Inc.

McKinlay, J., \& Marceau, L. (2004). Upstream healthy public policy: lessons from the battle of tabacco. In $\mathrm{C}$. Muntaner \& V. Navarro (Eds.). Political and Economic Determinants of Population Health and Well Being: Controversies and Developments. New York: Baywood Publishing Company

McKinlay, J., \& Marceau, L. (2000). To Boldly Go... American Journal of Public Health, 90, 25-33.

Mikkonen, J., \& Raphael, D. (2010). Social Determinants of Health: The Canadian Facts. Toronto: York University School of Health Policy and Management.

Montero, M., \& Varas-Díaz, N. (2007). Latin American Community Psychology. In S. M. Reich, M. Riemer, I. Prilleltensky \& M. Montero (Eds.). International community Psychology. History and Theories. (pp. 6398). New York: Springer.

Muntaner, C. \& Lynch, J. (2002). Social capital, class, gender and race conflict, and population health: An essay review of Bowling Alone's implications for social epidemiology. International Journal of Epidemiology, 31:261-267.

Muntaner, C., \& Navarro, V. (2004). Conclusion: Political, Economic, and Cultural Determinants of Population Health - A Research Agenda. In C. Muntaner \& V. Navarro (Eds.). Political and Economic Determinants of Population Health and Well Being: Controversies and Developments. New York: Baywood Publishing Company. International Journal of Epidemiology, 31, 261-267.

Nieves-Lugo, K., \& Toro-Alfonso, J. (2011). A description of health belief, social support, stress, and depression in the adherence behavior in a sample of men living with HIV in Puerto Rico. American Psychological Association 2011 Convention Presentation, $1-2$.

Navarro, V. (2009). What we mean by social determinants of health. Global Health Promotion, 16(1), 5-16.

Organización Mundial de la Salud. (2013). Estadísticas sanitarias mundiales 2013: Una mina de

información sobre salud pública mundial. Recuperado en http://www.who.int/gho/publications/world health_statistics/2013/es/index.html

Organización Mundial de la Salud, Europa. (2008). Determinantes Sociales de la Salud: Los Hechos Probados. Madrid: Ministerio de Sanidad y Consumo.

Organización Mundial de la Salud. (2003). Social Determinants of Health: The Solid Facts. (2nd Ed). R. Wilkinson \& M. Mamot (Eds). Author.

Organización Mundial de la Salud. (1998). Promoción de la Salud Glosario. Recuperado en: http://www.msssi.gob.es/profesionales/salu dPublica/prevPromocion/docs/glosario.pdf

Organización Mundial de la Salud. (1946). Constitución de la Organización Mundial de la Salud. Nueva York: Autor

Peterson, A., \& Lupton, D. (1996). The new public health: health and self in the age of risk. London: Sage Publications Ltd.

Polanco-Fronteras, N., Cajigas-Vargas, I., Rivera-Segarra, E., Santos-Figueroa, A., Rosario- Hernández, E. \& Varas-Díaz, N. (2013). El Estigma Relacionado a Problemas de Salud Mental: Resultados de un Grupo de Profesionales de la Salud en Adiestramiento en Puerto Rico. Salud $y$ Sociedad, 4 (3), 250-263.

Raphael, D. (2006). Social Determinants of Health: Present status, unanswered questions, and future directions. International Journal of Health Services, 36 (4), 651-677.

Rivera-Rivera, J., Rodríguez-Madera, S., \& Varas-Díaz, N. (2013). The Role of Social Support on Health-Related Practices of HIV-Discordant Heterosexual Couples. Revista Puertorriqueña de Psicología, 24 (2), 1-18.

Rivera-Segarra, E. \& Ramos-Pibernus, A. (2013). Stigma as a Social Determinant of Health: A Fundamental Cause Theory Perspective. In A. Trimboli, et al. (Eds.). 
Social Inclusion Trough Interdisciplinary Interventions (pp. 1027-1030). Buenos Aires: World Federation for Mental Health.

Saforcada, E., Lellis, M. \& Mozobancyk, S. (2010). Psicología y Salud Pública: Nuevos aportes desde la perspectiva del factor humano. Argentina: Editorial Paidós

Sermons, M. \& Witte, P. (2011). State of Homelessness in America: A Research Report on Homelessness. National Alliance to End Homelessness.

Tendenciaspr. (2010). Compendio de estadísticas: violencia en Puerto Rico. Proyecto tendenciaspr, Universidad de Puerto Rico, Recinto de Río Piedras. Recuperado

en: http://www.tendenciaspr.com.

Toro-Alfonso, J. \& Varas-Díaz, N. (2004). Los otros: prejuicio y distancia social hacia hombres gays y lesbianas en una muestra de estudiantes a nivel universitario. International Journal of Clinical and Health Psychology, 4 (3), 537-551.

Universidad de Puerto Rico, Recinto de Ciencias Médicas. (2012). Oferta Académica.http://www.rcm.upr.edu/PublicH ealth/Documentos/Boletin-Informativo.pdf

US Census Bureau (2008-2012). Poverty status in the past 12 months. American Community Survey 5-years estimate: Author.

Varas-Díaz, N., Malavé-Rivera, S. \& CintrónBou, F. (2008). AIDS stigma combinations in a simple of Puerto Rican health professionals: qualitative and quantitative evidence. Puerto Rico Health Sciences Journal 27 (2), 147- 157.

Varas-Díaz, N. \& Serrano-García, I. (2003). The challenge of a positive self-image in a Colonial context: A psychology of liberation for the Puerto Rican Experience. American Journal of Community Psychology, 31(1), 103-115.

Varas-Díaz, N., Santiago-Negrón, S., Neilands, T., Cintrón-Bou, F. \& Malavé-Rivera, S. (2010). Stigmatization of Illicit Drug use among Puerto Rican Health Professionals in Training. Puerto Rico Health Sciences Journal, 29 (2), 109-116.

Varas-Díaz, N., \& Toro-Alfonso, J. (2005). The stigmatization of HIVIAIDS and gregarious life: Contradictions in the development of social support networks for people living with HIVIAIDS in Puerto Rico. Science \& Behavior, 20, 111-142.

Venkatapuram, S., \& Marmot, M. (2009). Epdemiology and social justice in light of social determinants of health research. Bioethics, 23(2), 79-89. doi:10.1111/j.1467-8519.2008.00714.x

Wilkinson, R. \& Marmot, M. (2003). Social Determinants of Health: The Solid Facts. World Health Organization. 